\title{
Critical Pressure of Intramural Delamination in Aortic Dissection
}

\author{
Ehsan Ban, Cristina Cavinato, and Jay D. Humphrey \\ Department of Biomedical Engineering \\ Yale University, New Haven, CT, USA
}

Address for correspondence:

EB (ehsan.ban@yale.edu)

\begin{abstract}
Computational models of aortic dissection can provide novel insights into possible mechanisms by which this potentially lethal condition develops and propagates. We present results from a phase-field based finite element simulation of a classical experiment that had not previously been understood. Initial simulations agreed qualitatively and quantitatively with the experimental findings, but because of the complexity of the boundary value problem it was still difficult to build intuition. Hence, simplified analytical models were extended to gain further insight. Together, the simplified models and phase-field simulations revealed a power-law-based relation between the pressure required to initiate an intramural tear and key geometric and mechanical factors area of the initial insult, stiffness of the wall, and characteristic energy of tearing. The degree of axial stretch and luminal pressure similarly influenced the value of the tearing pressure, which was $~ 70 \mathrm{kPa}$ for a healthy aorta having a sub-millimeter-sized initial insult but even lower for larger tear sizes. Finally, the simulations showed that the direction a tear propagates can be altered by focal regions of weakening or strengthening, which can drive the tear towards the lumen (re-entry) or adventitia (rupture). Additional data are needed, however, on aortas having different predisposing disease conditions.
\end{abstract}

Keywords: delamination, phase field, intra-lamellar strength, tearing pressure 


\section{Introduction}

Aortic dissection is a life-threatening event that is increasingly responsible for significant morbidity and mortality. Despite considerable clinical experience, histo-pathological characterizations, in vitro biomechanical findings, and computational simulations, ${ }^{14,31,37}$ the precise mechanisms by which the aorta dissects remain unknown. It is axiomatic, however, that dissections arise when mechanical stresses exceed material strength, hence emphasizing the importance of performing and interpreting mechanical tests on the aorta. Not surprisingly, many different types of experiments have been reported, including uniaxial tests to failure, in-plane shearing tests, so-called peeling tests on strips of tissue, and pressurization to failure of cylindrical segments. Although peeling and in-plane shearing tests provide particularly important information on intra-lamellar adhesion strength (e.g., Refs. 25,32), these tests necessarily impose non-physiological loading conditions on a non-physiological geometry. Among others, Margot Roach and colleagues presented results from a clever experiment that preserved the native cylindrical geometry and in vivo relevant biaxial loading conditions while examining intra-lamellar adhesion - they used a 25-gauge needle to inject pressurized ink into the media of an excised but intact pressurized segment of the aorta. ${ }^{27}$ They found that the mean tearing pressure, defined as the difference between the imposed intra-lamellar pressure and the prescribed luminal pressure, was on the order of $547 \mathrm{mmHg}(\sim 73 \mathrm{kPa})$ while the mean pressure at which the dissection propagated was $54 \mathrm{mmHg}(\sim 7.2 \mathrm{kPa})$, independent of the depth within the media at which the tip of the needle rested. Because of the complexity of the mechanics associated with this experiment, these findings were discussed primarily in qualitative terms. Given the potential importance of the findings, there was a need to revisit these data and to seek increased understanding.

In this paper, we present a finite element based solution of the experiment reported by Roach and colleagues that provides greater insight into the many factors that dictate the associated propagation of a tear within the aortic wall. Notwithstanding the advantages of our phase field approach, the complexity of this three-dimensional boundary value problem yet rendered it initially difficult to gain broad intuition. Hence, we also constructed multiple highly idealized boundary value problems (using adherent beams, membranes, and plates) that reflect different aspects of the complex experiment of interest, thus increasing insight into both the mechanisms of failure and the different factors - degree of the initiating insult, wall stiffness, and fracture properties - that drive delamination of the aortic media. Finally, we examined computationally the potential impact of focal inhomogeneities of wall properties on the direction of the propagation. These findings provide some clues regarding both re-entry tears (i.e., dissections that turn inward toward the lumen) and transmural ruptures (i.e., dissections that turn outward toward the adventitia).

\section{Methods}


Finite element phase-field model of intra-lamellar tearing. Two- and three-dimensional finite element models were used to simulate the in vitro injection of ink into the medial layer of pressurized segments of excised intact aortas. ${ }^{27}$ Specifically, we used a phase-field finite element framework that we validated in a previous study of ink injection into cut-open, flat segments of aortic tissue. ${ }^{4}$ This phase-field model of tearing ${ }^{10}$ is based on a global minimization of energy. Tissue tearing is defined by a phase-field $\phi \in[0,1]$, where $\phi=0$ and 1 correspond to intact and fully damaged tissue.

In the three-dimensional model, the finite element mesh consisted of a cylindrical domain divided into $\sim 500,000$ tetrahedral elements, generated using an in-house script. The displacement field $\boldsymbol{u}$ was defined over the domain, and isochoric deformations were enforced using a Lagrange multiplier field $p$. Only a quarter of the vessel geometry was modeled, with zero displacements prescribed at one end of the cylindrical domain, as in the biaxial tests on intact segments, and symmetric boundary conditions on the other end. Residual stresses were accounted for naturally by prescribing constituent-specific deposition stretches via the elastic constitutive relation. ${ }^{30}$ These deposition stretches were 1.08 and 1.2 for each family of collagen fibers and for elastin, respectively. The two-dimensional model was generally similar, but focused on a single cross-section. In both cases, luminal pressure was applied as a traction boundary condition over the luminal surface (Fig. 1(a), (b)). The applied pressure at the current increment of loading remained normal to the deformed luminal surface, evaluated at the previous increment. During the computation, the luminal pressure was increased gradually from 0 to 130 $\mathrm{mmHg}$ to represent the mean arterial pressure prescribed experimentally. ${ }^{27}$ The initial model included a damaged volume that corresponded to the torn area created by the insertion of the needle. Injection of ink into the arterial wall was modeled by incrementally prescribing the volume of the ink, $V_{\text {injection, }}$ using a global Lagrange multiplier, $m .{ }^{4}$ Tears were modeled as concentrated regions of damage surrounded by elastic material, similar to previous analyses of rubber-like elastomers ${ }^{26}$ and arterial tissue. ${ }^{19}$

At the $j$ 'th increment of injection volume, the fields $\boldsymbol{u}_{j}, p_{j}, \phi_{j}$ and the scalar $m_{j}$ are determined by the alternate minimization of the energy

$$
E_{\text {total }}=E_{\text {deformation }}+E_{\text {tear }}+E_{\text {pressurized-fluid }} \text {. }
$$

$E_{\text {deformation }}$ denotes the energy due to deformation of the aortic wall, consisting of three contributions: the strain energy, a term that enforces isochoric deformations, and a regularization term. ${ }^{4} E_{\text {tear }}$ is the energy of tearing, that is, the characteristic energy of tearing, $G_{C}$, multiplied by a function of $\phi$ that represents the damaged area. ${ }^{4} G_{c}$ is the energy expended to extend the torn area by one unit. A value of $52 \mathrm{~J} / \mathrm{m}^{2}$ was used for $G_{C}$, which lies within the range of values previously reported. ${ }^{25,28,31}$ We incrementally prescribed the volume of injection by the minimization of $E_{\text {pressurized-fluid, }}$

$$
E_{\text {pressurized-fluid }}=m\left(\int_{V} \phi \lambda_{1} \lambda_{2} \lambda_{3} d V-V_{\text {injection }}\right) \text {. }
$$


Finally, we sought $\left(\boldsymbol{u}_{j}, p_{j}, m_{j}, \phi_{j}\right)=\underset{\boldsymbol{u}, p, m, \phi}{\operatorname{argmin}} E_{\text {total }}(\boldsymbol{u}, p, m, \phi)$ by iterative minimization by finding roots of the expressions $\nabla_{\bar{u}, \bar{p}, \bar{m}} E_{\text {total }}$ and $\nabla_{\bar{\phi}} E_{\text {total }}$, where $\nabla_{\bar{u}, \bar{p}, \bar{m}}$ denotes a directional derivative with respect to $\boldsymbol{u}, p$, and $m$ in the direction of their respective test functions. ${ }^{4}$ Similarly, $\nabla_{\bar{\phi}}$ denotes the directional derivative with respect to $\phi$ in the direction of the test function for $\phi$. The expressions $\nabla_{\bar{u}, \bar{p}, \bar{m}} E_{\text {total }}=0$ and $\nabla_{\bar{\phi}} E_{\text {total }}=0$ constitute the weak forms for the problem. The solution to the progressive damage problem was constrained between $\phi$ at the previous increment of injected volume and its maximum value of 1 , thus enforcing irreversible damage via tearing. We implemented the Bubnov-Galerkin method ${ }^{21}$ within the FEniCS finite element framework. ${ }^{2}$

Fung-type constitutive model of the arterial wall with four families of fibers. The native arterial wall was modeled using an incompressible hyperelastic constitutive relation that accounts for overall structural contributions of elastin, collagen, and smooth muscle. A neoHookean contribution to the strain energy models the deformation of the elastin-dominated amorphous material. Collagen fibers and passive smooth muscle were modeled as Fung-type exponentially strain-stiffening materials. ${ }^{30}$ The constitutive model is defined by the strain energy density function

$$
W_{\text {wall }}=\frac{\bar{c}}{2}\left(\lambda_{1}^{2}+\lambda_{2}^{2}+\lambda_{3}^{2}-3\right)+\sum_{i=1}^{4} \frac{c_{1}^{i}}{4 c_{2}^{i}}\left(\exp \left(c_{2}^{i}\left(\left(\lambda^{i}\right)^{2}-1\right)^{2}\right)-1\right)
$$

where $\bar{c}, c_{1}^{i}$, and $c_{2}^{i}$ are material constants. The principal stretch ratios are denoted by $\lambda_{1}, \lambda_{2}$, and $\lambda_{3}$, and the stretch ratios in the four main structural directions $i$ are denoted by $\lambda^{i}$, with $i=1$, 2,3 , and 4 corresponding to the axial, circumferential, and two symmetric diagonal directions, respectively. The diagonal directions lie on the axial-circumferential plane and are oriented at angles $\pm \alpha$ with respect to the axial direction. Illustrative material parameters were obtained from the data of Ref. 16 for the human descending thoracic aorta (Fig. 1(c)), as modeled in Ref. 30 , to increase clinical relevancy, noting that porcine (studied by Roach and colleagues ${ }^{27}$ ) and young human (considered herein) aortas exhibit similar material behaviors. ${ }^{6}$ The inner radius and thickness of the artery were $11.75 \mathrm{~mm}$ and $1.75 \mathrm{~mm}$, respectively. 

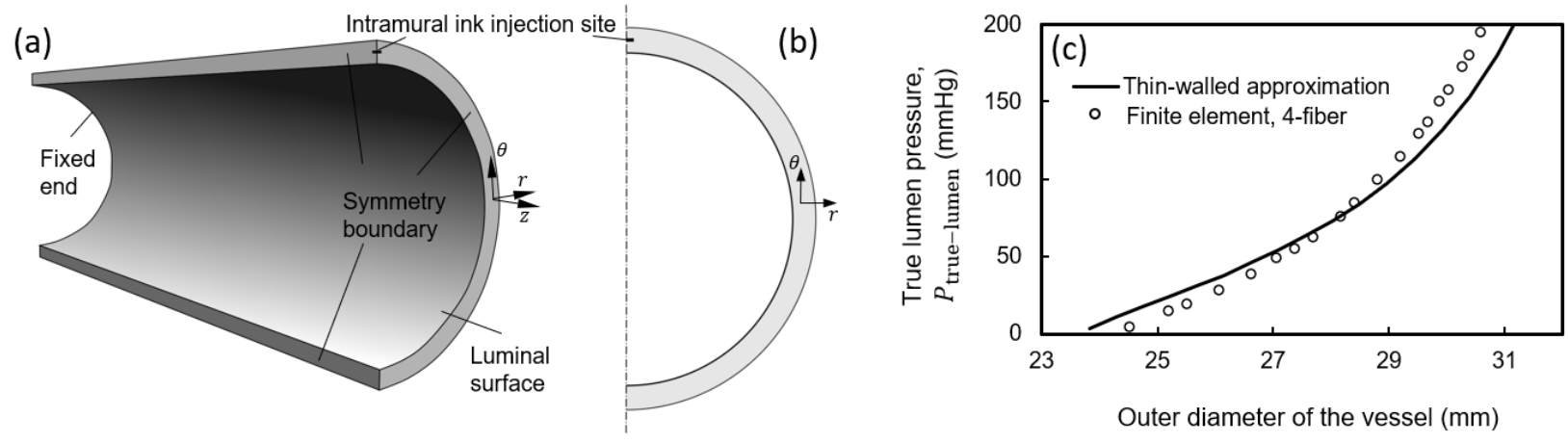

Figure 1. Model setup for propagating an intramural tear in a biaxially loaded artery by an intramural injection of ink. (a) Three-dimensional model of half of the arterial section, modeled using symmetric boundary conditions. Zero displacements were prescribed at the exposed surface of the pressurized and axially extended vessel at the fixed end, resembling a biaxial test setup, and symmetric displacement boundary conditions were prescribed at the other cross-sectional boundary surfaces. The volume of the damaged tissue was prescribed in increments beginning with the injection site, which models the incision made by the needle. (b) The two-dimensional model of injection into the aortic wall. Symmetric displacement boundary conditions were employed in this model as well. (c) Verification of the implementation of the three-dimensional constitutive relation in the finite element code by comparison with an analytical thin-walled approximation of wall deformation during biaxial loading of the artery. The pressure of the true lumen versus the outer diameter of the vessel at various pressures. An exponential Fung-type model with four families of fibers was used in both models.

Intramural injection into porcine aortic sections. To confirm experimentally the effect of the torn area on the pressure required for tearing, we repeated the experiments of Roach et al. $(1999)^{27}$ using needles of two different sizes, namely, 30- and 25-Gauge needles. Eighteen descending thoracic aortas of similar length $(56 \pm 3 \mathrm{~mm})$ were harvested from adult male Yorkshire pigs $(22-27 \mathrm{~kg})$, used in separate studies that did not affect the aortic mechanics and microstructure, in accordance with protocols approved by the Institutional Animal Care and Use Committee of Yale University. Perivascular tissue was removed by gentle dissection and all branches were ligated using nylon sutures. The samples were secured with ligatures on customdrawn acrylonitrile butadiene styrene cannulas, mounted within a custom biaxial testing system, and immersed in Hanks' Balanced Salt Solution (HBSS) throughout the duration of the test. The samples were then stretched axially to a stretch ratio of 1.1, which was also prescribed in our computational simulations. Following a short period of cyclic preconditioning (pressurization from 10 to $130 \mathrm{mmHg}$ with the vessel held fixed 1.1 axial stretch), the true lumen of the vessel was maintained at a pressure of $130 \mathrm{mmHg}$, equivalent to the mean arterial pressure in pigs that was prescribed by Roach and colleagues. ${ }^{27} \mathrm{~A} 1$-inch long $25 \mathrm{G}$ or $30 \mathrm{G}$ needle was inserted into the aortic wall in the direction of the longitudinal axis of the artery and at the minimum allowed angle to the external surface of the sample. The penetration depth was consistently $1 \mathrm{~mm}$ on the basis of the protruding length of the needle. In tests using the thicker needle, following insertion of the needle, we applied a small backward motion to withdraw the needle slightly to increase the 
initial volume of the damaged tissue while holding the end of the needle in the medial layer. A syringe pump was used to inject HBSS infused with India ink at the rate of $1 \mathrm{~mL} \mathrm{~min} \mathrm{~m}^{-1}$. The maximum pressure of injection was measured using a pressure transducer connected to the tubing that conveyed the injection fluid from the pump to the needle. The samples were randomly assigned into the $25 \mathrm{G}$ or $30 \mathrm{G}$ group. Statistical analysis of the data included comparison of the pressures of tearing for the two groups using the Student's t-test.

\section{Results}

Tears propagate suddenly after reaching a threshold injection pressure. The threedimensional computational model included a damaged area representative of the initial tear made by inserting the needle. As the volume of the injected ink increased, the damaged area initially grew larger and deformed the surrounding tissue without propagating the initial tear. Then, at a critical pressure of fluid, $P_{\text {tear }}$, the tear started to propagate, accompanied by a reduction in injection pressure (Fig. 2(a)). Upon further increase in the volume of injection, the tear propagated farther, increasing the torn area of the tissue (Fig. 2(b) and (c)). The tear propagated in both axial and circumferential directions, creating biconcave volumes. Both the injection pressure versus volume curves and the morphology of the vessels agreed well with those reported by Roach et al. (1999). ${ }^{27}$
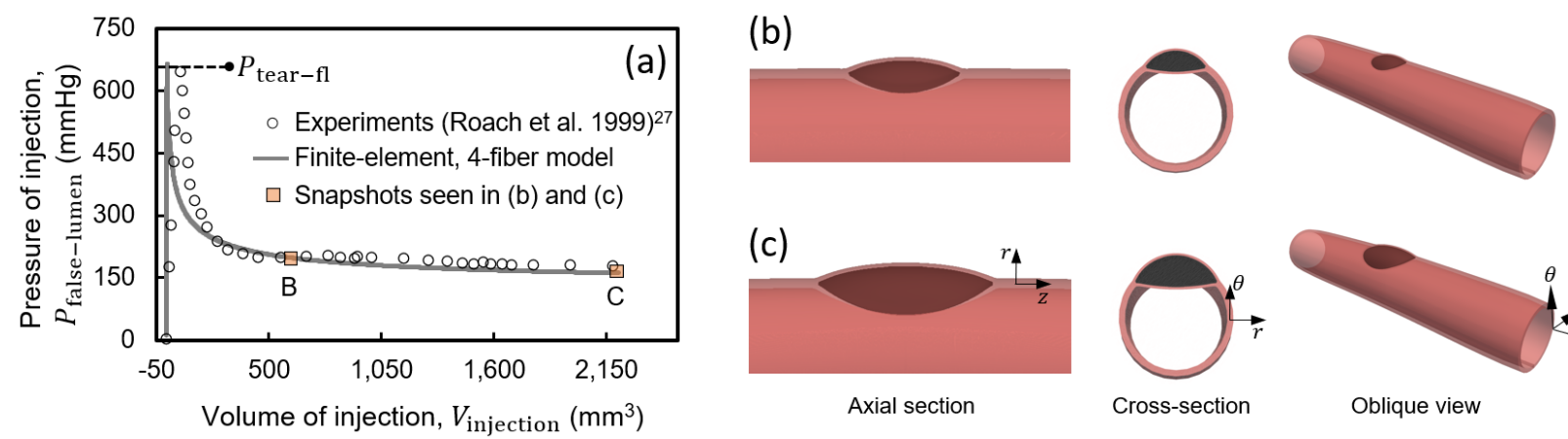

(c)
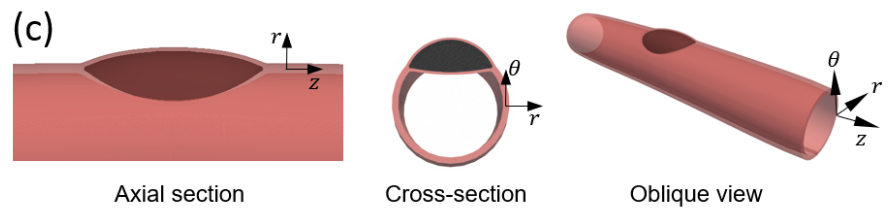

Oblique view

Figure 2. Finite element simulations of the intramural injection of ink within the media of a biaxially loaded vessel modeled by a Fung-type constitutive law with four families of fibers. (a) As ink was injected into the arterial wall, the pressure of injection initially increased rapidly while deforming the surrounding tissue. After reaching a critical value of pressure, $P_{\text {tear }}$, the tear started to propagate axially and then circumferentially. Both the (a) injection pressure-volume curves and (b) the morphology of the injected volume agree with those reported by Roach et al. (1999). ${ }^{27}$

\section{Minimalistic analytical models of pressure-separation of adhered linear elastic} structures. We also developed simple extensions of previous analytical models ${ }^{17}$ of two interleaved tissue flaps and two fluid pools to gain further insights into the pressure of tearing in the false lumen relative to the pressure of the true lumen as well as its dependence on the properties of the tear and the arterial wall. We previously used similar models ${ }^{17}$ to study delamination of cut-open aortic tissue by injection, but the effects of a counter-acting true lumen 
pressure was absent in these models. That motivated us to extend the previous models. The extended models included initially adherent linearly elastic structures that were separated by the action of the pressures applied to model true and false lumens (i.e., pressures applied outside of and between the structures, respectively). We derived such relations for initially adherent linearly elastic slender beams, circular membranes, and circular plates (Fig. 3(a)-(g)) - see Appendix A for details. Together, these models suggested that the pressure of tearing of the false lumen $\left(P_{\text {tear-fl }}\right)$ and the pressure of the true lumen $\left(P_{\text {true lumen }}\right)$ relate as

$$
P_{\text {tear-fl }}^{\eta}+\left(P_{\text {tear-fl }}-P_{\text {true-lumen }}\right)^{\eta}=c^{2} \propto \frac{K_{t}^{\alpha} G_{c}^{\beta}}{A_{\text {torn }}^{\gamma}},
$$

where $c$ is related to the resistance of the tear to propagation, $K_{t}$ is related to the tangent elastic stiffness, $G_{C}$ is the characteristic energy of tearing, and $A_{\text {torn }}$ is the torn area of the tissue. The exponents $\alpha, \beta$, and $\gamma$ take positive values but vary depending on the elastic structure in the model. In the linear models with beams, plates, or highly tense membranes, $\eta=2$ and at constant resistance of the tear, $c$, Eq. 4 thus describes the relationship between $P_{\text {tear-fl }}$ and $P_{\text {true-lumen }}$ as the equation of an ellipse (curves in Fig. 3(h)).

Detailed interpretation of these models is not recommended because of the use of linear elastic models. Nonetheless, qualitative insights emerge for intramural tearing of the wall by intramural injection. In the regime where $P_{\text {tear-fl }}>P_{\text {true-lumen }}$ (shaded area in Fig. 3(h)), the false lumen has a biconcave morphology (Fig. 3(h), inset) and $P_{\text {tear-fl }}$ increases with increasing $P_{\text {true-lumen. }}$. The needle injection experiments lie in this region. In contrast, if $P_{\text {tear-fl }}<$ $P_{\text {true-lumen, }}$ the false lumen has a meniscus-like morphology (Fig. 3(h), inset) and $P_{\text {tear-fl }}$ decreases with increasing $P_{\text {true-lumen. }}$ In the regime where $P_{\text {tear-fl }}>P_{\text {true-lumen }}$ and its vicinity, the ellipse-shaped curve of the minimal model, Eq. 4, agrees reasonably well with results from finite element computations using a simplified material model, a neo-Hookean model. The main observation based on the analytical models is qualitative and limited to the regime of the injection experiments where the pressure of the false lumen is larger than the pressure of the true lumen. In this regime, the critical pressure of tearing decreases with increasing size of the false lumen. Similarly, the critical pressure of tearing increases with increasing tissue stiffness and characteristic energy of tearing. Importantly, these minimalistic models suggest power-law relations that were thus investigated further based on full nonlinear finite element models below. 
(a)

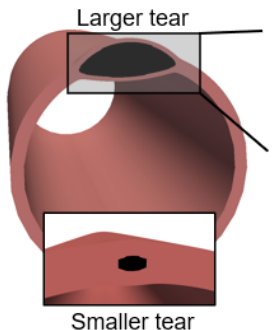

(d)

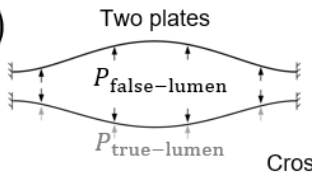

(f)

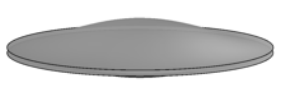

(b)

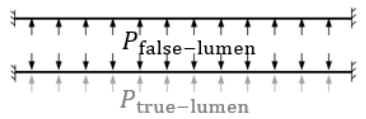

(c)

Oblique view

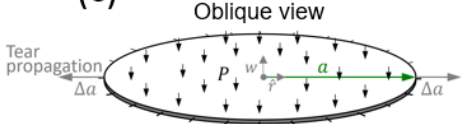

(e)

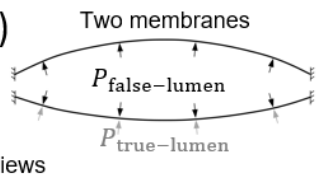

(g)

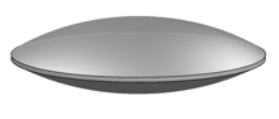

Oblique views

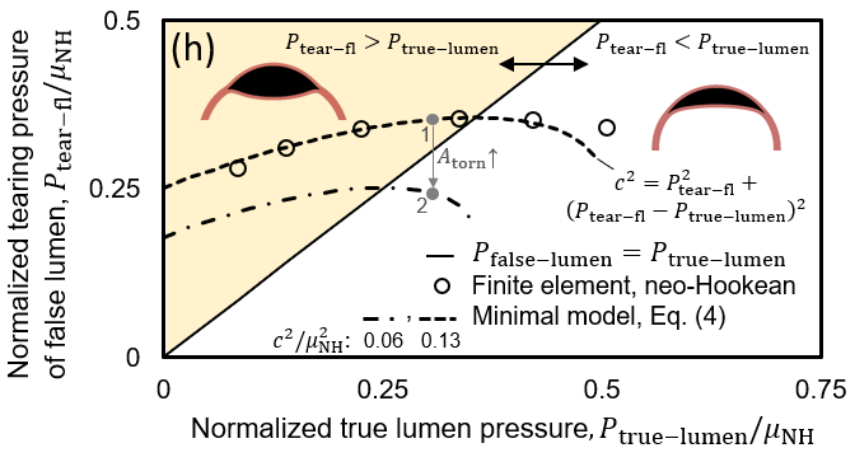

Figure 3. Schematic views of extensions of three minimalistic analytical models ${ }^{17}$ of the separation of adhered linear elastic structures with comparison to a neo-Hookean finite element simulation. (a) Snapshot from the finite element model of intramural injection. A tear bigger than the initial torn area in Fig. 2 is shown to highlight the tissue deformation and tearing by an exaggerated view. The initial insults made by a needle appears substantially smaller prior to the start of tear propagation. (b and c) Crosssectional and oblique views of the loading of two portions of the wall by an intramural pressure, $P_{\text {false-lumen }}$ and pressure in the true lumen, $P_{\text {true-lumen }}$ in the minimalistic models. The models consist of initially adherent beams, circular plates, or circular membranes with zero displacements prescribed at their ends / periphery. ( $d$ and e) Cross sectional and ( $f$ and $g$ ) oblique schematic views of the deformation of the two ( $d$ and $f$ ) plates or ( $e$ and $g$ ) membranes. The highly simplified linear models suggested that the pressure of tearing at the false lumen relates to the pressure of the true lumen via an equation of an ellipse, $P_{\text {tear-fl }}^{2}+\left(P_{\text {tear-fl }}-P_{\text {true-lumen }}\right)^{2}=c^{2} \propto K_{t}^{\alpha} G_{c}^{\beta} / A_{\text {torn }}^{\gamma}$ (h) Comparison of the results from a minimal model with a finite element model of a pressurized cylinder modeled as a simplified neo-Hookean solid with an initial tear. The solid line indicates when pressures in the two lumens equal. Above the line, where $P_{\text {false-lumen }}>P_{\text {true-lumen, }}$, the larger pressure in the false lumen pushes the septum into the true lumen and leads to a biconcave shape (insets). Below the line, the pressure in the true lumen is larger, which leads to a crescent or meniscus shape of the false lumen. The trend of the slopes of the ellipses change at their intersection with the $P_{\text {false-lumen }}=P_{\text {true-lumen }}$ line. $P_{\text {tear-fl }}$ increases with increasing $P_{\text {true-lumen }}$ above the line; the reverse is observed below the line. The gray arrow shows the path going from point 1 to 2 with an increase in the original torn area of the wall. Interpretation of the model is limited to the regime of the injection experiments, where the pressure of the false lumen is larger than 
that of the true lumen. These qualitative results are examined below using nonlinear finite element models of the arterial wall described by a Fung type 4-fiber model.

The pressure of tearing of the false lumen depends nonlinearly on the pressure of the true lumen, vessel stiffness, characteristic energy of tearing, and in vivo axial stretch. To probe changes of $P_{\text {tear-fl }}$ with vessel properties, we performed computations using the fully nonlinear phase-field finite element model with a four-fiber Fung-type constitutive law. First, as the fixed pressure of the true lumen was increased incrementally from 0 to $130 \mathrm{mmHg}, P_{\text {tear-fl }}$ showed an increasing trend. After that, it reached a plateau (Fig. 4(a)). Similarly, $P_{\text {tear-fl }}$ decreased with decreases in the fixed value of axial stretch of the biaxially loaded vessel (Fig. 4(a), inset). As expected, $P_{\text {tear-fl }}$ increased with increases in both the characteristic energy of tearing of the aortic wall and its native tangent stiffness (Fig. 4(b) and (c)). The exponents from Eq. (4) that relate tear resistance to $K_{t}$ and $G_{c}$ were found to be $\alpha=1.0$ and $\beta=1.5$. 


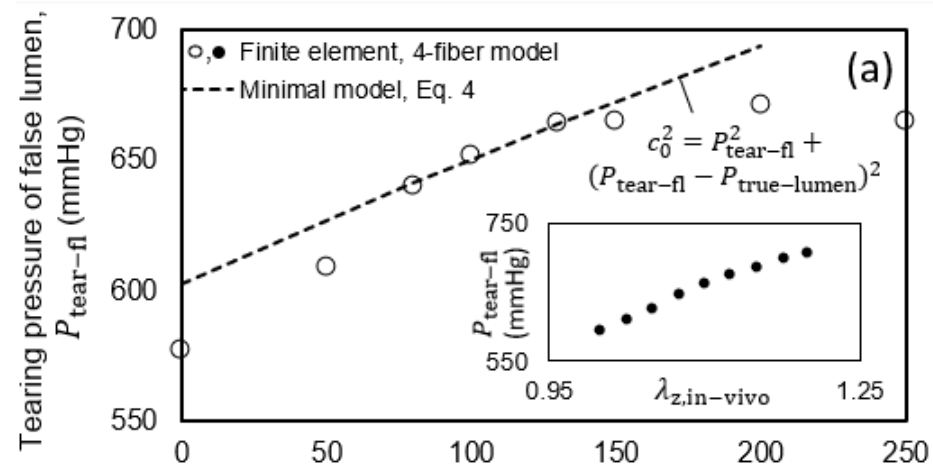

True lumen pressure, $P_{\text {true-lumen }}(\mathrm{mmHg})$
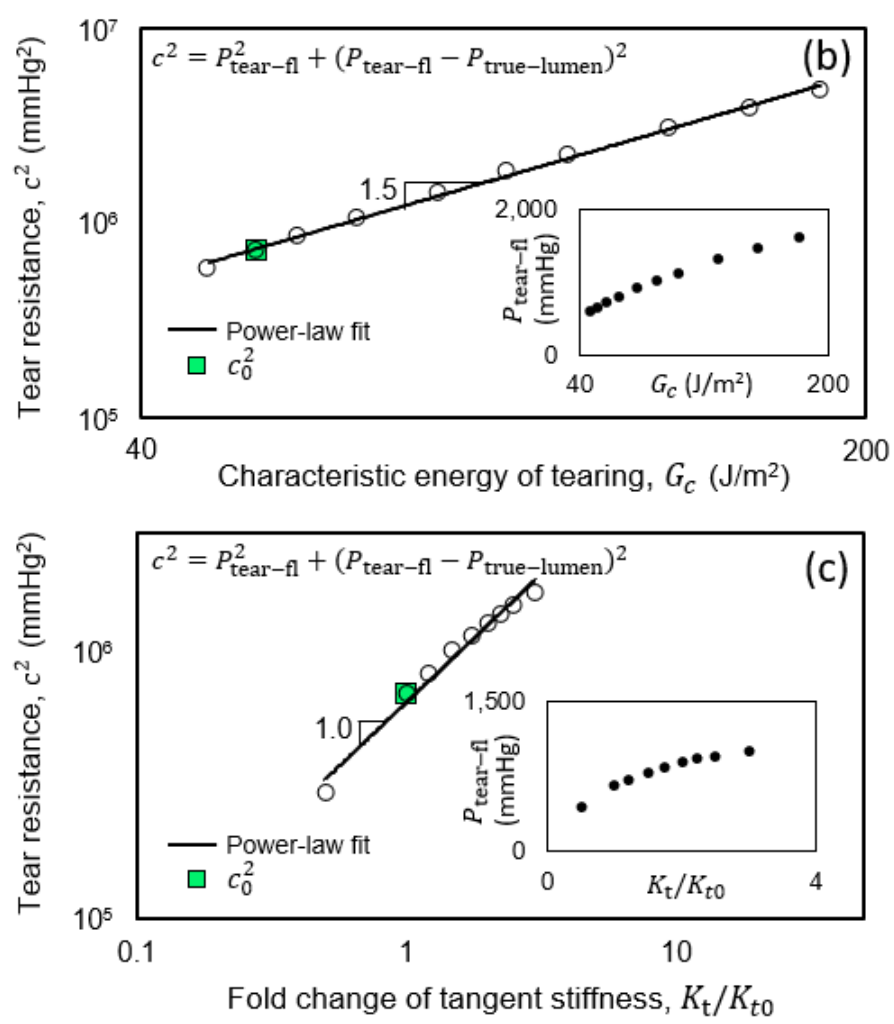

Figure 4. Dependencies of $P_{\text {tear-fl }}$ on luminal pressure with tear properties assessed by the fully nonlinear finite element model employing a Fung-type wall model with four families of fibers. (a) Variation of the pressure of tearing of the false lumen with the pressure of the true lumen. The dashed curve shows the fit to an equation of an ellipse, $c_{0}^{2}=P_{\text {tear-fl }}^{2}+\left(P_{\text {tear-fl }}-P_{\text {true-lumen }}\right)^{2}$, motivated by the minimalistic analytical models of linearly elastic materials. $c_{0}^{2}$ in this curve corresponds to the point $P_{\text {true-lumen }}=130 \mathrm{mmHg}$, the mean arterial pressure in the experiments of Roach and colleagues. Change of $P_{\text {tear-fl }}$ with changing axial stretch of the vessel is plotted in the inset. (b and c) Dependence of tear resistance, $c^{2}=P_{\text {tear-fl }}^{2}+\left(P_{\text {tear-fl }}-P_{\text {true-lumen }}\right)^{2}$, on changing tissue properties; $G_{c}$ and $K_{\mathrm{t}} / K_{t 0}$ denote the characteristic energy of tearing and fold change of tangent stiffness of the tissue. The solid lines are power-law fits to the data. Changes of $P_{\text {tear-fl }}$ with $G_{c}$ and $K_{\mathrm{t}} / K_{t 0}$ are plotted in the insets. In panels (b) and (c), all axes of the main plots are logarithmic. 
The pressure of tear progression reduces with increasing size of the initial torn area. To assess effect of the initial area of tissue damage (due to the insertion of a needle or possible in vivo defects) on the pressure required for tear propagation, we performed similar finite element computations using various values of $A_{\text {torn }}$. In the first set of tests, the torn area was prescribed isotropically (circles in Fig. 5(a)), whereas, in the second set, it had an elongated shape (triangles in Fig. 5(a)). In both sets of experiments, $P_{\text {tear-fl }}$ decreased with increasing values of $A_{\text {torn }}$. We also measured the torn area as the tear propagated, starting from a small $A_{\text {torn, }}$ using the pressure values in Fig. 2. The exponent $\gamma$ (Eq. 4), which relates tear resistance to $A_{\text {torn }}$ was evaluated as $0.5,0.6$, and 0.7 for cases with isotropic and elongated changes of $A_{\text {torn }}$ and the case of propagation, starting from a small $A_{\text {torn }}$. Our additional experiments showed a decrease of $P_{\text {tear-fl }}$ with increasing needle size $(p$-value $=0.01)$ as the needle gauge changed from 30 to 25 , increasing the size of the initial tear made by inserting the needle. The resulting experimentally measured change of $P_{\text {tear-fl }}$ with $A_{\text {torn }}$ agreed qualitatively with results from the nonlinear finite element model using the four-fiber Fung type model.
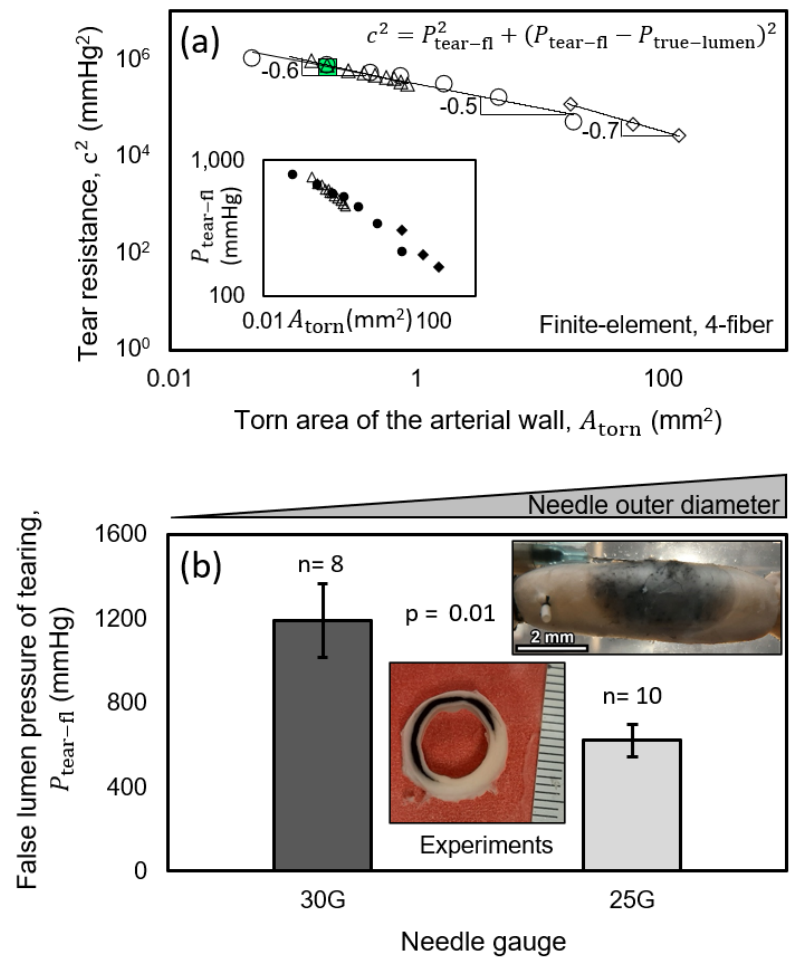

Figure 5. The pressure of tearing of the false lumen decreases with increases in the initial torn area. (a) The nonlinear finite element model based on a Fung-type four-fiber family model suggests that $P_{\text {tear-fl }}$ decreases with increases in the initial torn area. Note that tear resistance squared, $c^{2}=P_{\text {tear-fl }}^{2}+$ $\left(P_{\text {tear-fl }}-P_{\text {true-lumen }}\right)^{2}$. The circles correspond to models with isotropic changes of the area, triangles correspond to elongated torn areas, and diamonds correspond to the area recorded during tear propagation in Fig. 2. Variation of $P_{\text {tear-fl }}$ with $A_{\text {torn }}$ is shown in the inset. All axes in panel (a) were plotted on a logarithmic scale. (b) The pressure of tearing was larger in tests with an initial torn area 
created by a thinner needle ( $p$-value $=0.01$ ). Needles of gauges 25 and 30 were tested. Axial and crosssectional snapshots of the injection experiments are shown in the top and bottom insets.

Tear propagation follows oblique regions of weakness of the wall. We used the finite element model to assess possible deflection of the path of tear propagation by inhomogeneities. In the two-dimensional model with homogeneous wall properties, the tear consistently propagated circumferentially (Fig. 6(a)). We considered localized changes in wall composition / organization that modeled areas of focal aberrant material accumulation (strengthening) or degradation (weakening), and thus strong inhomogeneities, including local accumulations of mucoid material, calcification, and fibrosis. Regions of weakening had values of tangent stiffness and characteristic energies of tearing that were reduced by a factor of three in comparison with the surrounding media. Regions of strengthening had tangent stiffnesses and characteristic energies of tearing five times that of the surrounding media. Tests with both radially oriented weakening and strengthening inhomogeneities suggested that tear propagation remained unchanged in the presence of radially oriented inhomogeneities. The tears passed through the inhomogeneities and continued to propagate in the circumferential direction (Fig. 6(b) and (c)). Tests with oblique weakenings showed, however, that the path of propagation of a tear may be deflected by these inhomogeneities. Focal regions of weakness oriented at angles of roughly 15 degrees with respect to the circumferential direction were able to guide the tear to propagate inward toward the true lumen or outward toward the outer surface of the vessel (Fig. 6(d) and (e)). A strong oblique inhomogeneity could similarly deflect the path of tear propagation (Fig. $6(f))$. Taken together, these results demonstrate the possibility that the path of propagation of a tear can be influenced dramatically by inhomogeneities in wall properties.
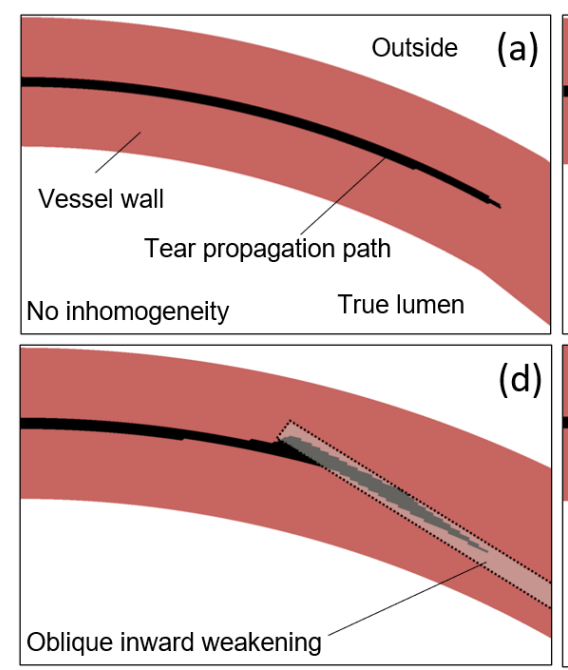
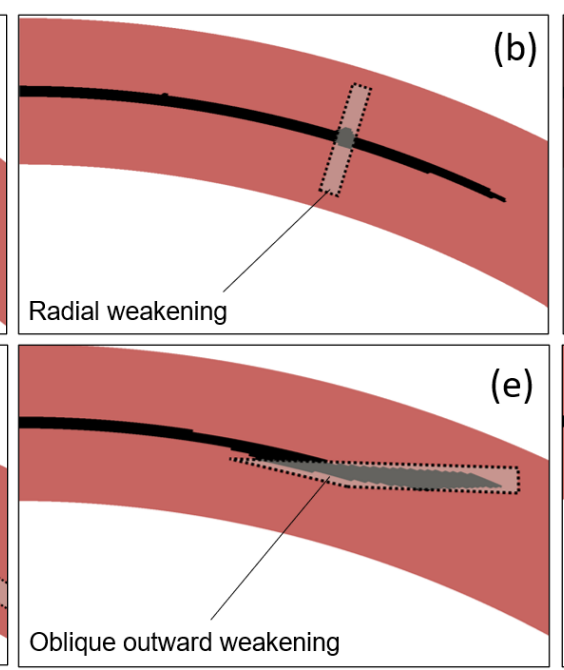

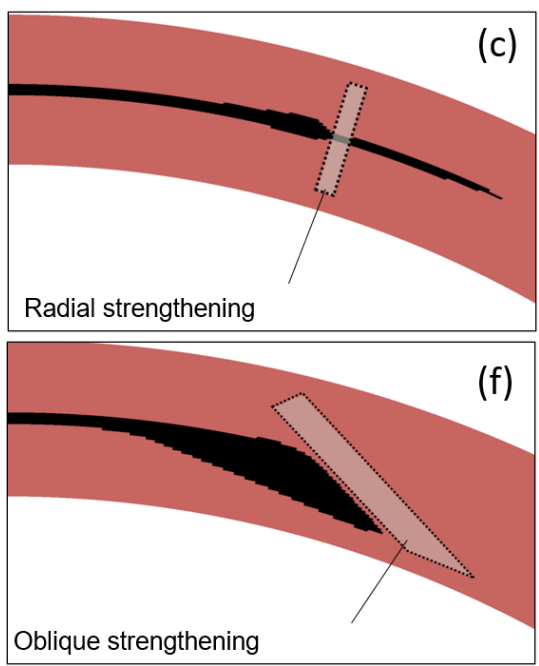

Figure 6. Inhomogeneities of wall properties can direct the path of propagation of a tear from the circumferential direction, guiding the tear toward the true lumen or alternatively toward the outside surface of the vessel. (a) The tear tended to propagate circumferentially in a homogeneous arterial wall. (b and c) The tear similarly propagated circumferentially in the presence of either a radially oriented 
weakening or strengthening inhomogeneity. ( $d$ and e) An oblique region of weakness of the wall oriented at roughly 15-degree angles with respect to the circumferential direction could deflect the path of propagation toward the true lumen or outside of the vessel. (f) A strengthening inhomogeneity oriented at 30 degrees with respect to the circumferential direction could also deflect the propagation of an inhomogeneity. In panels (b), (c), and (f), there are build-ups of the injected fluid in the vicinity of the tear before the tear goes into a stiffer and stronger portion of the vessel wall.

\section{Discussion}

Margot Roach and colleagues reported two particularly important experimental studies of aortic dissection in the 1990s but noted that the associated "mechanical analysis is complex." 12,27 In both cases, they quantified the pressure required to initiate and propagate an intralamellar delamination within the porcine aorta independent of prior disease. We previously showed that a phase-field finite element model was able to capture the experimental findings reported in 1990 for excised slabs of aorta into which ink was injected under pressure. ${ }^{4}$ That study revealed region-specific mechanisms of aortic delamination as well as potential power-law relations that govern the propagation of a dissection, but it was unclear whether these relations depended strongly on the nearly flat in vitro geometry that was used experimentally and simulated computationally. In this paper, we found that our basic phase-field finite element approach captured similar data reported in 1999 for a more physiological geometry (cylindrical) and quasi-static biaxial loading (including the initial residual stress field). As in the experiments, the simulated pressure required to initiate the tear was much higher than that required to propagate the tear. The simulations revealed further that this pressure of tearing increases with acute increases in luminal pressure and axial stretch. Importantly, the in vivo value of axial stretch often diminishes in vessels having a propensity to dissect. ${ }^{7}$

Again, power-law relations emerged for the pressure at which the tear begins to propagate in terms of (proportionally) local wall stiffness and the characteristic energy of tearing and in terms of (inversely) the initial area of the tear. We submit that this power-law relation (Eq. 4), supported by simple linear models and confirmed via nonlinear finite element methods, helps to clarify and summarize what are otherwise complex nonlinear effects and effectors. Such relations have promise to consolidate data from many different experiments on vessels having different vulnerabilities, a continuing experimental need. Finally, we also found that localized regions of intramural weakening or strengthening can affect the direction of the propagation, possibly turning an otherwise preferential circumferential propagation (Fig. 5(b)) towards either the lumen (possibly driving a re-entry tear) or the adventitia (possibly predisposing to transmural rupture). These findings point to the importance of improved in vivo imaging capability to assess risk of propagation of a pre-existing dissection, particularly with regard to rupture potential. Collectively, these present results confirm the importance of material properties as well as the type and size of the initial insult and the external loading conditions. Identifying the precise mechanisms of pathogenesis remains challenging, though intimal tears ${ }^{24,34}$ and focal intramural 
accumulations of highly negatively charged mucoid material ${ }^{22,29}$ are potential initiators. At the nucleation of the tear, when the size is below a millimeter, a normal blood pressure is too low to separate the aortic layers and advance the tear. The swelling pressure of mucoid materials, however, is sufficient to initiate the separation of the elastic lamella. ${ }^{1,29}$ With increasing volume of the false lumen, the concentration of the charges decreases, resulting in a reduction in swelling pressure. The inverse relation between the critical pressure required for tearing and the size of the false lumen suggests the possibility of the continuation of delamination of larger false lumen either by elevated blood pressure or the progressive accumulation of mucoid material at higher concentrations. Continuing focus on intra-lamellar structure and strength similarly promises to provide continued insight. ${ }^{24,36}$

Notwithstanding the advantages of the present study, there are numerous limitations. First, Roach and colleagues studied healthy porcine aortas, hence limiting the relevancy of the specific values of the tearing pressure. Vascular aging, uncontrolled hypertension, and genetic predispositions (e.g., Marfan syndrome and Loeys-Dietz syndrome) often precede aortic dissections, and mouse models have revealed that each of these conditions associates with significant changes in aortic composition and material properties. ${ }^{7,9,15}$ There is a need to repeat the experiments of Roach and colleagues and associated computational simulations in vessels excised from vulnerable human samples or animals that model these key predisposing factors. Although $60 \%$ of aortic dissections occur independent of aneurysm, ${ }^{20}$ there is similarly a need to repeat the present analysis in vessels that are aneurysmal since they also associate with marked changes in aortic composition and properties, which can vary locally. ${ }^{8}$ That said, perhaps the greatest challenge, both experimentally and computationally, is the need to delineate the precise focal versus global changes in the wall that likely initiate dissection. ${ }^{3}$ Exquisite imaging of the microstructure of the vulnerable aorta followed by pressurization tests under in vivo conditions is likely the best way forward.

In summary, previous computational models (including those based on partition of unity and extended finite elements) have provided considerable insight into the biomechanics of aortic dissection ${ }^{1,19,33,35,39}$ as well as the importance of the effects of solid-fluid interactions. ${ }^{5,13,23}$ In this paper, we showed further that phase-field based finite element models are able to capture key experimental findings and to reveal important new power-law relations that promise to aid in understanding further the biomechanical mechanisms of initiation and propagation of aortic dissection.

\section{Acknowledgments}

We thank the Sinusas Lab at Yale University for providing us with aortic tissue samples. This work was supported, in part, by a grant from the US National Institutes of Health (U01 HL142518). 
Appendix A: Pressure of tearing in analytical models of two adhered linearly elastic plates or membranes. In our previous study of region-specificity of dissections ${ }^{4}$, we used mathematical relationships amongst critical pressure, stiffness, and characteristic energy of tearing proposed by Gent and Lewandowski $(1987)^{17}$ to model delamination by injection of cut-open arterial segments. To use similar mathematical relations in the case of an inflated artery we made simplified models that extend the analysis of Gent and Lewandowski (1987) ${ }^{17}$ to include two flaps of tissue interleaved by two pools of pressurized fluid (Fig. A1 and Fig. 3). The pressure of the false lumen deforms the two flaps of tissue while the pressure of the true lumen resists the deformation and the deformation of the four elements are coupled.

We employed an energy-based approach ${ }^{18}$ to find the critical pressure required to separate two adhered linear elastic circular plates or membranes in the presence of a counteracting fluid pressure acting over the outer surface of one of the circular elements (Fig. 3 (b) and (c)). In this minimal model, we neglect the deformation of the artery outside of the circular areas. The solid bodies share the same radius $a$, thickness, $h$, elastic modulus, $E$, and Poisson's ratio, $v$. The energy required to advance the torn surface by unit area is $G_{c}$. The energy-based approach requires evaluation of the total energy that is comprised of the energies of the elastic deformation of the inner and outer flaps of tissue, $U_{\text {inner }}$ and $U_{\text {outer }}$, the energy loss by the pressure in the true and false lumens, $-P_{\mathrm{tl}} \Delta V_{\mathrm{tl}}$ and $-P_{\mathrm{fl}} \Delta V_{\mathrm{fl}}$, and finally the energy of tearing $\pi a^{2} G_{c}$. The volumes of the true and false lumens and the deformation of the flaps interact such that $\Delta V_{\text {fl }}=V_{\text {out-flap }}+V_{\text {in-flap }}$, with $\Delta V_{\mathrm{tl}}=-V_{\text {in-flap }} . V_{\text {out-flap }}$ and $V_{\text {in-flap }}$ are the volumes of fluid enclosed by the deformed outer and inner flaps. In comparison with the energy in the finite element implementation, instead of prescribing the injection volume, here, we consider the state of deformation and tearing due to application of a constant pressure at the false lumen. The stationary point of the total energy with respect to the tear size determines the pressure of tearing.

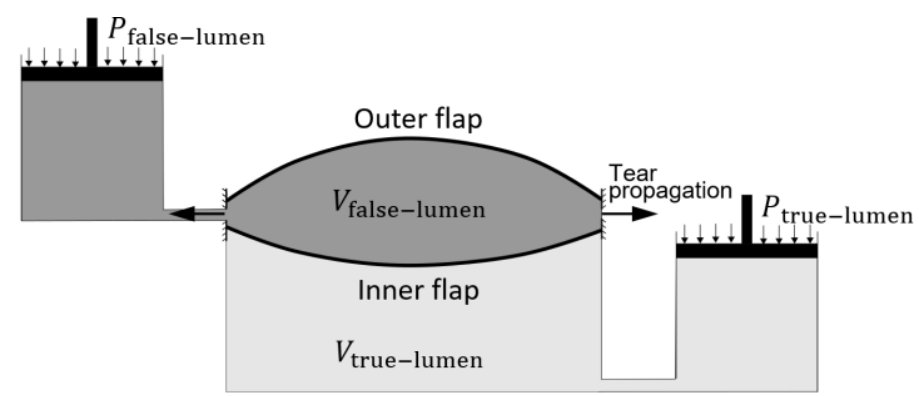

Figure A1. Schematic of the deformation of the inner and outer flaps of the aortic wall by the pressure of the true lumen and the intramural pressure of injection in the false lumen. The pressure of the false lumen deforms the outer flap of tissue while the differential of pressure of the true and false lumens deforms the inner flap. The interaction of the two pools of fluid is modeled by the loss of the volume of false lumen as the volume of the true lumen increases and vice versa. 
In the case of the small deformation of a single linearly elastic plate, the axisymmetric displacement of the thin plate, $w(\hat{r})$, at a radius $\hat{r}$ is governed by $\nabla^{2} \nabla^{2} w(\hat{r})=P / B . P$ is the total uniformly distributed pressure and $B=E h^{3} /\left(12\left(1-v^{2}\right)\right)$ is the bending stiffness of the plate. Given the prescribed zero displacement at the periphery of the plate (Fig. 3(c)) and the non-zero displacement in the middle of the plate, $w(\hat{r})=P\left(\hat{r}^{2}-a^{2}\right)^{2} /(64 D)$. If we consider that initially, the torn area is negligible, the energy required to produce a torn area of radius $a$ is $E_{\text {tear }}=$ $\pi a^{2} G_{c} \cdot{ }^{38}$ The volume of fluid enclosed by the deforming plate is $V=\int_{A} w(\hat{r}) d \mathrm{~A}$, where $A$ is the circular area of the plate before deformation. The elastically stored energy in the plate is $U=$ $a^{6} P^{2} \pi\left(1-v^{2}\right) /\left(32 E h^{3}\right)$.

The outside plate, located between the false lumen and the outside surface of the vessel is loaded by the pressure from the false lumen, $P=P_{\text {tear-fl }}$, whereas, the inside plate, sandwiched between the true and false lumens, is loaded by the differential of pressure of the two $P=P_{\text {tear-fl }}-P_{\text {true-lumen. }}$. Following the approach of Griffith (1921), ${ }^{18}$ the critical pressure of tearing corresponds to the tear size at the stationary point of $E_{\text {total }}$, i.e., where $\partial E_{\text {total }} / \partial a=$ 0 . Therefore, in the case of two-plates

$$
P_{\text {tear-fl }}^{2}+\left(P_{\text {tear-fl }}-P_{\text {true-lumen }}\right)^{2}=\frac{32 E G_{c} h^{3}}{3 a^{4}\left(1-v^{2}\right)}=c^{2}
$$

For the tear to advance between two deforming membranes interleaved by two pools of fluid, we extend the analysis of Gent and Lewandowski (1987) ${ }^{17}$ for a single membrane. The change of potential of the fluid pressure is $-P V$. The deformation of the membrane may be characterized by approximate relations for the maximum deflection of the membrane, $\delta$, and the volume of the fluid enclosed by the membrane: $\delta=C_{2}\left(P a^{4} /(E h)\right)^{1 / 3}$ and $V=C_{1} \pi a^{2} \delta$. Since $P \propto V^{3}$, and $U=\int_{V} P d V, U=P V / 4$ in case of the nonlinear deformation of the membrane. By repeating the same steps as in the case of two plates, we obtain the relationship

$$
P_{\text {tear-fl }}^{4 / 3}+\left(P_{\text {tear-fl }}-P_{\text {true-lumen }}\right)^{4 / 3}=\frac{2.6 G_{c} E^{1 / 3} h^{1 / 3}}{a^{4 / 3}} .
$$

A linear solution for highly tensed membranes ${ }^{11}$ results in the relationship

$$
P_{\text {tear-fl }}^{2}+\left(P_{\text {tear-fl }}-P_{\text {true-lumen }}\right)^{2}=\frac{8 G_{c} h \sigma}{a^{2}}=c^{2},
$$

where $\sigma$ is the initial stress in the membranes.

Finally, if we consider two elastic beams with rectangular sections, instead of two plates, and repeat this approach, with vanishing deflection and angles at the end of the beams, we obtain the following relationship 


$$
P_{\text {tear-fl }}^{2}+\left(P_{\text {tear-fl }}-P_{\text {true-lumen }}\right)^{2}=\frac{3 G_{c} E h^{3}}{2 a^{4}}=c^{2} .
$$

\section{References}

1. Ahmadzadeh, H., M. K. Rausch, and J. D. Humphrey. Modeling lamellar disruption within the aortic wall using a particle-based approach. Sci. Rep. 9:15320, 2019.

2. Alnæs, M., J. Blechta, J. Hake, A. Johansson, B. Kehlet, A. Logg, C. Richardson, J. Ring, M. E. Rognes, and G. N. Wells. The FEniCS Project version 1.5. Archive of Numerical Software 3, 2015.

3. Aslanidou, L., M. Ferraro, G. Lovric, M. R. Bersi, J. D. Humphrey, P. Segers, B. Trachet, and N. Stergiopulos. Co-localization of microstructural damage and excessive mechanical strain at aortic branches in angiotensin-II-infused mice. Biomech. Model. Mechanobiol. 19:81-97, 2020.

4. Ban, E., C. Cavinato, and J. D. Humphrey. Differential propensity of dissection along the aorta. Biomech. Model. Mechanobiol. 20:895-907, 2021.

5. Bäumler, K., V. Vedula, A. M. Sailer, J. Seo, P. Chiu, G. Mistelbauer, F. P. Chan, M. P. Fischbein, A. L. Marsden, and D. Fleischmann. Fluid-structure interaction simulations of patient-specific aortic dissection. Biomech. Model. Mechanobiol. 19:1607-1628, 2020.

6. de Beaufort, H. W. L., A. Ferrara, M. Conti, F. L. Moll, J. A. van Herwaarden, C. A. Figueroa, J. Bismuth, F. Auricchio, and S. Trimarchi. Comparative analysis of porcine and human thoracic aortic stiffness. Eur. J. Vasc. Endovasc. Surg. 55:560-566, 2018.

7. Bellini, C., M. R. Bersi, A. W. Caulk, J. Ferruzzi, D. M. Milewicz, F. Ramirez, D. B. Rifkin, G. Tellides, H. Yanagisawa, and J. D. Humphrey. Comparison of 10 murine models reveals a distinct biomechanical phenotype in thoracic aortic aneurysms. J. R. Soc. Interface 14:20161036, 2017.

8. Bersi, M. R., C. Bellini, J. D. Humphrey, and S. Avril. Local variations in material and structural properties characterize murine thoracic aortic aneurysm mechanics. Biomech. Model. Mechanobiol. 18:203-218, 2019.

9. Bersi, M. R., R. Khosravi, A. J. Wujciak, D. G. Harrison, and J. D. Humphrey. Differential cellmatrix mechanoadaptations and inflammation drive regional propensities to aortic fibrosis, aneurysm or dissection in hypertension. J. R. Soc. Interface 14:20170327, 2017.

10. Bourdin, B., G. A. Francfort, and J.-J. Marigo. The variational approach to fracture. J. Elasticity 91:5-148, 2008.

11. Campbell, J. D. On the theory of initially tensioned circular membranes subjected to uniform pressure. Q. J. Mech. Appl. Math. 9:84-93, 1956.

12. Carson, M. W., and M. R. Roach. The strength of the aortic media and its role in the propagation of aortic dissection. J. Biomech. 23:579-588, 1990.

13. Dillon-Murphy, D., A. Noorani, D. Nordsletten, and C. A. Figueroa. Multi-modality imagebased computational analysis of haemodynamics in aortic dissection. Biomech. Model. Mechanobiol. 15:857-876, 2016. 
14. Dingemans, K. P., P. Teeling, A. C. van der Wal, and A. E. Becker. Ultrastructural pathology of aortic dissections in patients with Marfan syndrome: Comparison with dissections in patients without Marfan syndrome. Cardiovasc. Pathol. 15:203-212, 2006.

15. Ferruzzi, J., D. Madziva, A. W. Caulk, G. Tellides, and J. D. Humphrey. Compromised mechanical homeostasis in arterial aging and associated cardiovascular consequences. Biomech. Model. Mechanobiol. 17:1281-1295, 2018.

16. García-Herrera, C. M., D. J. Celentano, M. A. Cruchaga, F. J. Rojo, J. M. Atienza, G. V. Guinea, and J. M. Goicolea. Mechanical characterisation of the human thoracic descending aorta: experiments and modelling. Comput. Method. Biomec. 15:185-193, 2012.

17. Gent, A. N., and L. H. Lewandowski. Blow-off pressures for adhering layers. J. Appl. Polym. Sci. 33:1567-1577, 1987.

18. Griffith, A. A. VI. The phenomena of rupture and flow in solids. Philos. Trans. A Math. Phys. Eng. Sci. 221:163-198, 1921.

19. Gültekin, O., S. P. Hager, H. Dal, and G. A. Holzapfel. Computational modeling of progressive damage and rupture in fibrous biological tissues: application to aortic dissection. Biomech. Model. Mechanobiol. 18:1607-1628, 2019.

20. Guo, D.-C., E. S. Regalado, C. Minn, V. Tran-Fadulu, J. Coney, J. Cao, M. Wang, R. K. Yu, A. L. Estrera, H. J. Safi, S. S. Shete, and D. M. Milewicz. Familial thoracic aortic aneurysms and dissections. Circ. Cardiovasc. Genet. 4:36-42, 2011.

21. Hughes, T. J. R. The Finite Element Method: Linear Static and Dynamic Finite Element Analysis. Dover Publication, Mineola, New York, 2012.

22. Humphrey, J. D. Possible mechanical roles of glycosaminoglycans in thoracic aortic dissection and associations with dysregulated TGF- $\beta$. J. Vasc. Res. 50:1-10, 2013.

23. Keramati, H., E. Birgersson, J. P. Ho, S. Kim, K. J. Chua, and H. L. Leo. The effect of the entry and re-entry size in the aortic dissection: a two-way fluid-structure interaction simulation. Biomech. Model. Mechanobiol. 19:2643-2656, 2020.

24. Pal, S., A. Tsamis, S. Pasta, A. D’Amore, T. G. Gleason, D. A. Vorp, and S. Maiti. A mechanistic model on the role of "radially-running" collagen fibers on dissection properties of human ascending thoracic aorta. J. Biomech. 47:981-988, 2014.

25. Pasta, S., J. A. Phillippi, T. G. Gleason, and D. A. Vorp. Effect of aneurysm on the mechanical dissection properties of the human ascending thoracic aorta. J. Thorac. Cardiovasc. Surg. 143:460-467, 2012.

26. Rivlin, R. S., and A. G. Thomas. Rupture of rubber. I. Characteristic energy for tearing. J. Polym. Sci. 10:291-318, 1953.

27. Roach, M. R., J. C. He, and R. G. Kratky. Tear propagation in isolated, pressurized porcine thoracic aortas. Can. J. Cardiol. 15:569-575, 1999.

28. Roach, M. R., and S. H. Song. Variations in strength of the porcine aorta as a function of location. Clin. Invest. Med. 17:308-318, 1994.

29. Roccabianca, S., G. A. Ateshian, and J. D. Humphrey. Biomechanical roles of medial pooling of glycosaminoglycans in thoracic aortic dissection. Biomech. Model. Mechanobiol. 13:1325, 2014.

30. Roccabianca, S., C. A. Figueroa, G. Tellides, and J. D. Humphrey. Quantification of regional differences in aortic stiffness in the aging human. J. Mech. Behav. Biomed. 29:618-634, 2014. 
31. Sherifova, S., and G. A. Holzapfel. Biomechanics of aortic wall failure with a focus on dissection and aneurysm: A review. Acta Biomater. 99:1-17, 2019.

32. Sommer, G., S. Sherifova, P. J. Oberwalder, O. E. Dapunt, P. A. Ursomanno, A. DeAnda, B. E. Griffith, and G. A. Holzapfel. Mechanical strength of aneurysmatic and dissected human thoracic aortas at different shear loading modes. J. Biomech. 49:2374-2382, 2016.

33. Thunes, J. R., J. A. Phillippi, T. G. Gleason, D. A. Vorp, and S. Maiti. Structural modeling reveals microstructure-strength relationship for human ascending thoracic aorta. J. Biomech. 71:84-93, 2018.

34. Tong, J., Y. Cheng, and G. A. Holzapfel. Mechanical assessment of arterial dissection in health and disease: Advancements and challenges. J. Biomech. 49:2366-2373, 2016.

35. Wang, L., S. M. Roper, N. A. Hill, and X. Luo. Propagation of dissection in a residuallystressed artery model. Biomech. Model. Mechanobiol. 16:139-149, 2017.

36. Wang, R., X. Yu, and Y. Zhang. Mechanical and structural contributions of elastin and collagen fibers to interlamellar bonding in the arterial wall. Biomech. Model. Mechanobiol. 20:93-106, 2021.

37. Weinsaft, J. W., et al. Aortic dissection in patients with genetically mediated aneurysms: incidence and predictors in the GenTAC registry. J. Am. Coll. Cardiol. 67:2744-2754, 2016.

38. Williams, M. L. The continuum interpretation for fracture and adhesion. J. Appl. Polym. Sci. 13:29-40, 1969.

39. Yu, X., B. Suki, and Y. Zhang. Avalanches and power law behavior in aortic dissection propagation. Sci. Adv. 6:eaaz1173, 2020. 